\title{
Sight to Touch: 3D Diffeomorphic Deformation Recovery with Mixture Components for Perceiving Forces in Robotic-Assisted Surgery
}

\author{
Angelica I. Aviles ${ }^{1}$, Samar M. Alsaleh ${ }^{2}$ and Alicia Casals ${ }^{1}$
}

\begin{abstract}
Robotic-assisted minimally invasive surgical systems suffer from one major limitation which is the lack of interaction forces feedback. The restricted sense of touch hinders the surgeons' performance and reduces their dexterity and precision during a procedure. In this work, we present a sensory substitution approach that relies on visual stimuli to transmit the tool-tissue interaction forces to the operating surgeon. Our approach combines a 3D diffeomorphic deformation mapping with a generative model to precisely label the force level. The main highlights of our approach are that the use of diffeomorphic transformation ensures anatomical structure preservation and the label assignment is based on a parametric form of several mixture elements. We performed experimentations on both ex-vivo and in-vivo datasets and offer careful numerical results evaluating our approach. The results show that our solution has an error measure less than $1 \mathrm{~mm}$ in all directions and an average labeling error of $2.05 \%$. It can also be applicable to other scenarios that require force feedback such as microsurgery, knot tying or needle-based procedures.
\end{abstract}

\section{INTRODUCTION}

Robotic surgical systems are widely used nowadays to perform minimally invasive procedures as they enhance the performance of surgeons by providing motion scaling, tremor filtering and enhanced degrees of freedom at the instrument tips [1]. Patients favor the option of undergoing a Robotic-Assisted Minimally Invasive Surgery (RAMIS) over conventional options due to the associated benefits of reduced surgical trauma, faster recovery, and improved cosmetics [1]. However, currently available commercial surgical systems still suffer from an unresolved problem, which is the lack of haptic feedback to the operating surgeon [2], [3]. During RAMIS, surgeons loose the direct sense of touch creating a high mental workout for them as they need to infer the tooltissue interaction forces [4]. This limitation is considered an active field of research in medical robotics and is reputed to be one of the causes that restricts their further spread [2].

To retrieve the force information, many works have been focused on the design of force sensing devices that can be attached to the surgical instrument (see for example [5], [6], [7]). Nonetheless, those devices can also measure irrelevant force data, from the surgical environment [8], and/or are difficult to adapt to the surgical system due to different factors including: biocompatibility and sterilization constrains, longterm stability, size and high cost [9]. For these reasons, other

\footnotetext{
${ }^{1}$ A.I. Aviles and A. Casals are with the Center of Research of Biomedical Engineering Universitat Politècnica de Cataluya, Spain. E-mail: \{angelica.ivone.aviles, alicia.casals\}@upc.edu

${ }^{2}$ S.M. Alsaleh is with the Department of Computer Science, George Washington University, Washington DC, U.S.A. E-mail: sm57@gwu.edu
}
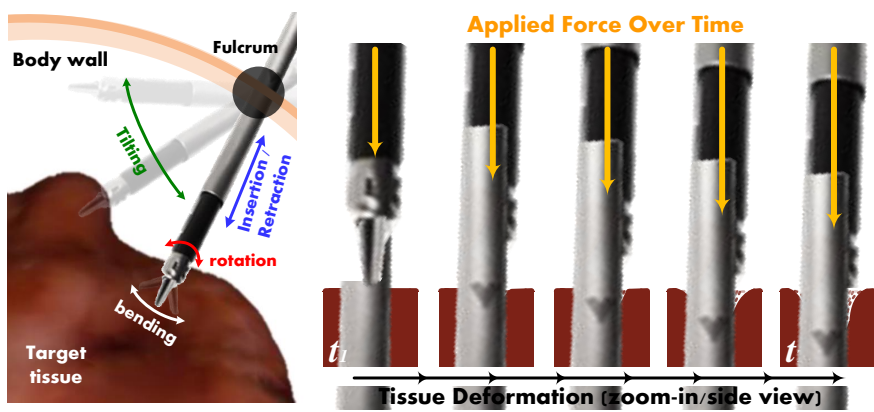

Fig. 1. Left side shows common surgical tasks while the right side illustrates how tissue deformation is directly proportional to the force applied over time.

research groups focus their efforts on utilizing the visual information that exists in a surgical system to estimate the interaction forces.

The feasibility of Vision-Based Force Estimation (VBFE) has been demonstrated in different scenarios such as in [10], [11], [12], [13]. Some works improve the VBFE solutions by incorporating soft computing, Artificial Neural Networks in particular, to achieve more accurate estimations of the interaction forces like in [14], [15], [16], [17].

After estimating the force, the resulted information can be transmitted to the operating surgeon through a sensory modality to provide a feedback of the forces interacting with the telemanipulated robot. Common sensory substitution modalities include auditory, vibrotactile, and visual cues [18]. Out of these options, visual feedback has the most advantages and is the most suitable for clinical adoption [8]. Various studies have investigated the feasibility of visual feedback on conveying force information for surgeons while performing delicate tasks and the results show improved performance among novice surgeons while decreasing inconsistencies [19].

Since all RAMIS settings include a videoscopic view of the operation, we can employ the available visual information of the tool-tissue interaction and relate it directly to the applied force (illustration in Fig. 1). From the conservation principles of continuum mechanics it is clear that the change in shape of an elastic object is directly proportional to the force applied. Following this principle, we propose a novel approach to perceive the interaction forces. Unlike our work presented in [16], here we optimize our variational framework that we use to compute the observable deformation after a force is applied. The main difference is twofold. First, we integrated a topology preservation term that guarantees 


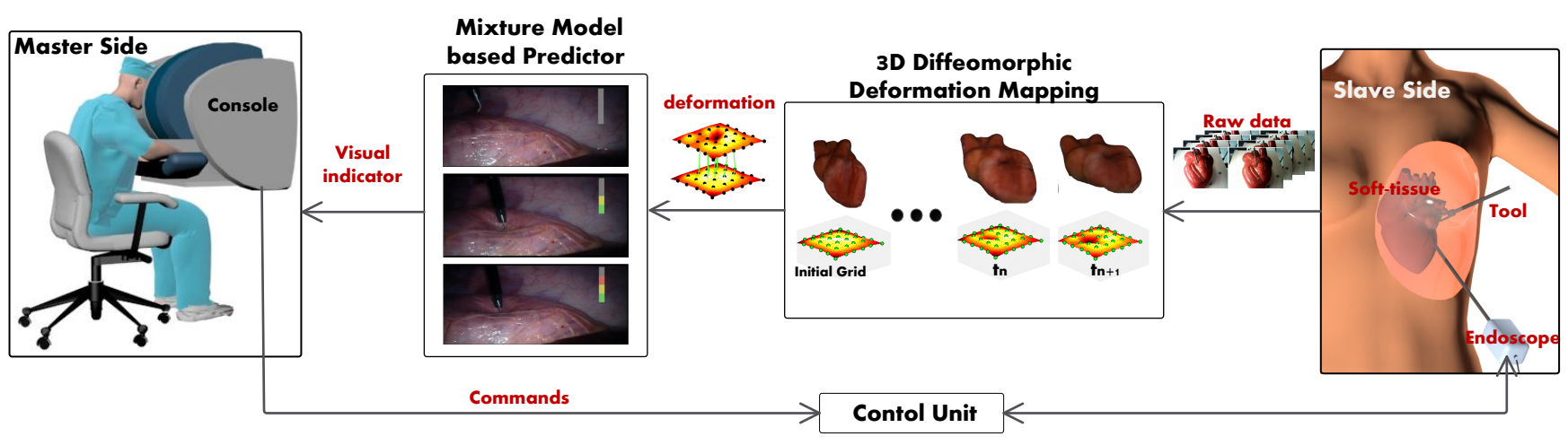

Fig. 2. Overall view of our approach for perceiving interaction forces in robotic surgical systems. We first compute a visual based approach that allows recovering the deformation structure over time. Then, deformation mapping is used to display the level of the applied force.

diffeomorphic transformation. Second, we use unsupervised learning approach that models the results in a parametric form of several mixture elements, resulting in an assignment of the deformation to a perceived force. While this is an important part of the solution, our main contributions are:

- We propose a 3D deformation mapping approach that guarantees a diffeomorphic transformation. It offers different advantages such as finding a better minima, decreasing the computational time and preserving the anatomical structure of the tissue.

- The exploration of a generative model to offer the surgeon a visual stimuli as a force feedback.

To the best of our knowledge, there is no previous report in the literature that combines a diffeomorphic deformation and a generative model to perceive the interaction forces.

\section{PERCEIVING THE INTERACTION FORCES}

In this section, we present our approach to perceive the interaction forces (see Fig. 2). This section contains two parts: first we describe our variational framework to compute the 3D diffeormophic deformation. Then we explain how the interaction forces are perceived.

\section{A. 3D Diffeomorphic Deformation Mapping}

Consider a stereo-pair image sequence, the left and right view as $f_{l}^{t}: \Omega_{l} \rightarrow \mathbb{R}^{2}$ and $f_{r}^{t}: \Omega_{r} \rightarrow \mathbb{R}^{2}$ where each image $f_{l}^{t}$ and $f_{r}^{t}$ is a function on the bounded domain of the corresponding $\Omega$ at time $t=1, \ldots, T$. With the aim of finding a trade-off between computational time and accuracy, we handle the tissue deformation using the following definition:

Definition 1: A $m$-dimensional lattice is a discrete subgroup of an Euclidean vector space with $k$-linearly independent vectors in $\mathbb{R}^{m}$.

Let $L_{l} \subset \mathbb{R}^{2}$ and $L_{r} \subset \mathbb{R}^{2}$ be the lattices defined at each view in a stereo pair image. Then, the three-dimensional lattice, $L \subset \mathbb{R}^{3}$, is computed from the projections of the corresponding points in the given lattices (see Fig. 3). The resulting $L$ is composed of a set of $v$ points, $\mathbf{P}$, where $\mathrm{p}_{v}=\left(y_{1}, \ldots, y_{m}\right)$. As a deformation model, we use the tensor product of the b-splines as they allow performing fast computations while keeping optimal mathematical properties [20]. Consider a given position $\mathbf{w}=\left(w_{1}, \ldots, w_{d}\right)$ in $\mathbb{R}^{d}$ and let $\xi(\cdot)$ be the basis spline function of degree $n$, then, the deformation at a given point $\mathbf{w}$ can be expressed as:

$$
\Phi(w ; \mathbf{P})=\sum_{j_{1}=0}^{n} \ldots \sum_{j_{d}=0}^{n} \mathbf{P}_{j_{1}, \ldots, j_{d}} \prod_{k=1}^{d} \xi_{k}\left(w_{k}\right)
$$

Taking Eq. 1, we now can compute the changes produced on the tissue over $t$ by minimizing the energy functional, $\mathrm{E}_{T}$, with respect to $\mathbf{P}$ as:

$$
\begin{aligned}
\mathbf{E}_{T}^{t}(\mathbf{P})= & \mathbf{E}_{d}\left(f_{l}^{t}(\Phi(w ; \mathbf{P})+w), f_{r}^{t}(w)\right) \\
& +\gamma \mathbf{E}_{r}(\Phi(w ; \mathbf{P}))+\mathbf{E}_{v p}(\Phi(w ; \mathbf{P}))
\end{aligned}
$$

where $E_{d}$ is the discrepancy term, $\mathbf{E}_{r}$ is the regularizer for obtaining a plausible transformation with the parameter $\gamma>0$, and $\mathbf{E}_{v p}$ is the constrain for preserving the anatomical structure.

In particular, for this work we selected as a discrepancy term the Sum of Squared Differences (SSD) together with a maximum likelihood type estimator. In this case, the minimization $\sum_{i} r^{2}$ of the residual error $r$ is changed by the Tukey's estimator expressed as $\rho$. We restrict the space of search using the Tikhonov regularizer. Our functional is then expressed as:

$$
\begin{aligned}
\mathbf{E}_{T}^{t}(\mathbf{P})= & \int_{\Omega} \rho\left(\left(f_{l}^{t}(\Phi(w ; \mathbf{P})+w)-f_{r}^{t}(w)\right)\right) d \Omega \\
& +\gamma \sum_{i=1}^{d} \int_{\Omega}\|\nabla \Phi(w ; \mathbf{P})\|^{2} d \Omega+\int_{\Omega} \delta_{\Phi}(w ; \mathbf{P}) d \Omega
\end{aligned}
$$

A particular advantage of our proposal is that we achieve a diffeomorphic deformation according to the next definition:

Definition 2: Given a smooth map, $f: X \rightarrow Y$ is a diffeomorphism if it is one-to-one and onto and also if $f^{-1}$ is smooth.

Guaranteeing diffeomorphic transformations is important to avoid violations of region convexity or distortion of the mesh elements, which results in an unrealistic deformation. In the case of small deformations, the smoothness of the regularizer can be enough but this is not the case when complex deformations appears. 


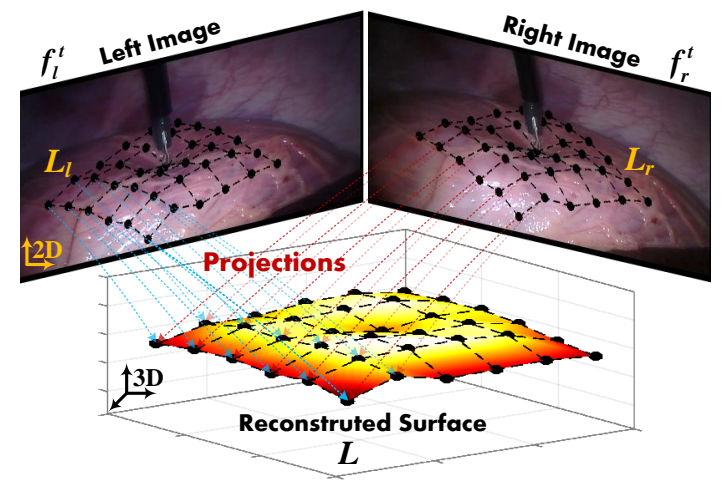

Fig. 3. 3D Diffeomorphic surface reconstruction from the projection of the lattice points defined at each stereo-pair image.

Now, we turn to explaining how we guarantee a diffeomorphic deformation by monitoring the Jacobian determinant $\left|J_{\Phi}(x: \mathbf{P})\right|$ of the deformation. To do this, we use a function that we first proposed in [21] in which the main difference is the non-trivial optimization process in the three dimensional space. From Eq. $3 \delta_{\Phi}$ is defined as:

$$
\begin{aligned}
\delta_{\Phi}(w ; \mathbf{P}) & := \begin{cases}\frac{\frac{1}{2} \pi-\arctan \left(\left|J_{\Phi}(w ; \mathbf{P})\right|\right)}{\pi}+\varphi \sqrt{\left|J_{\Phi}(w ; \mathbf{P})\right|^{2}} \text { if }(\star) \\
0\end{cases} \\
(\star)|| J_{\Phi}(w ; \mathbf{P})|-1| \geq \tau & \text { otherwise }
\end{aligned}
$$

where $\varphi>0$ offers a control fit in our penalization, and $\tau>0$ is the margin of acceptance for values close to one. To minimize Eq. 3, we use the Levenberg-Marquardt (LM) Algorithm.

\section{B. Labeling Assignment Approach}

Unlike most of the works in the state of the art (for example see [5], [6]) in which the interaction forces are directly estimated, in this work, we use the deformation mapping (as explained in subsection II-A) for creating a visual stimuli of the applied forces.

We transmit that stimuli by means of a dynamic bar at the corner of the display. The bar alternates between four different color indicators that correspond to how much force is applied. We assign a coding color based on the perceptual phenomena involved in color:

- Risk-free: is represented by the green color and reflects a minimal tool-tissue interaction

- Minimal Risk: symbolizes a safe amount of force and is coded as yellow

- Potential Risk: makes reference of a potential damage to the tissue and is coded as orange

- Risk: is indicated in red color to warn the physician of a tissue damage

The process of identifying where each observation belongs is given by modeling them in a parametric form of several mixture components and then assign them to each indicator based on its posterior probability. To do this, and on the basis of a training set, consider the set of points $\mathbf{P}$ at a given position $w$ as $\boldsymbol{x}=\left\{x^{(1)}, \ldots, x^{(L)}\right\}$ in a form $L \times D$,
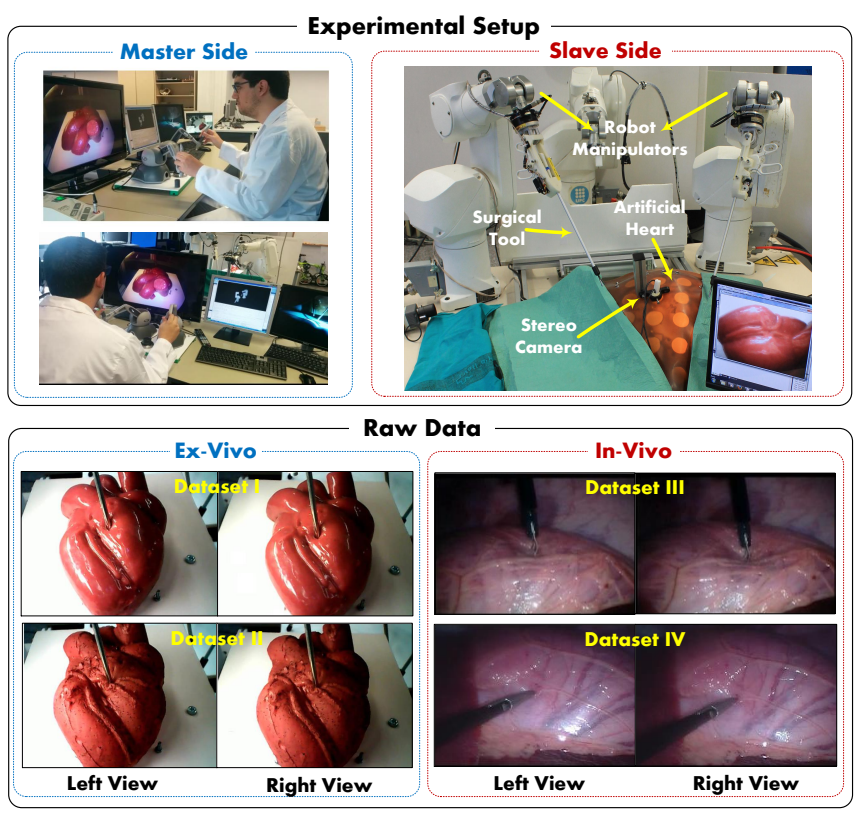

Fig. 4. (From top to bottom) The experimental setup used to acquire our ex-vivo datasets. Samples from the raw in-vivo and ex-vivo datasets used to evaluate our proposed solution.

then, we can model the $k$-component by maximizing the likelihood function as:

$$
\ln p(\boldsymbol{x} \mid \boldsymbol{\theta})=\sum_{l=1}^{L} \ln \left\{\sum_{k=1}^{K} \pi_{k} g\left(x_{l} \mid \theta_{k}\right)\right\}
$$

where $\boldsymbol{\theta}=\left\{\pi, \mu, \sum\right\}$ such that $\mu$ and $\sum$ are the mean and the covariance matrices respectively and $\pi$ is the mixture coefficients satisfying $\sum_{k=1}^{K} \pi_{k}=1$. Moreover, let $g$ be a $D$-dimensional multivariate gaussian density function expressed as:

$$
g\left(x_{l} \mid \theta_{k}\right)=\frac{1}{(2 \pi)^{D / 2}\left|\sum_{k}\right|^{-\frac{1}{2}}} e^{\frac{1}{2}\left(x-\mu_{k}\right)^{\top} \Sigma_{k}^{-1}\left(x-\mu_{k}\right)}
$$

From Eqs. 5-6, the objective is to find the set of $K$ parameters $\boldsymbol{\theta}=\left\{\pi_{1}, \mu_{1}, \sum_{1}, \ldots, \pi_{K}, \mu_{K}, \sum_{K}\right\}$ such that we can have an assignment for each deformation mapping. To find $\hat{\theta}=\operatorname{argmax}\{\ln p(\boldsymbol{x} \mid \boldsymbol{\theta})\}$, we use the ExpectationMaximization algorithm (EM) [22]. Let $\boldsymbol{z}=\left\{z^{1}, \ldots, z^{L}\right\}$ be the $L$ labels associated with the $l-t h$ sample (given lattice points), then the marginal likelihood is found by iterating, until a local maxima, using the Expectation and Maximization steps (E-step and M-step) as:

- E-step: Computes the expectation of the log-likelihood function given $\boldsymbol{x}$ and the current estimate $\hat{\theta}^{t}$ as: $Q\left(\boldsymbol{\theta}, \hat{\theta}^{t}\right)=E\left[\ln p\left(\boldsymbol{x}, \boldsymbol{z} \mid \theta^{t}\right) \mid \boldsymbol{x}, \theta^{t}\right]$

- M-step: Updates the estimate using current estimates as: $\theta^{t+1}=\operatorname{argmax}_{\theta}=Q\left(\boldsymbol{\theta}, \hat{\theta}^{t}\right)$

As mentioned before, Eqs. 5-6 are computed on a training dataset. Once $\boldsymbol{\theta}$ is found, we use it to predict the label of coming data. The advantages of formulating the recovered deformation as mixture elements is the computational tractability and handling uncertainty. 

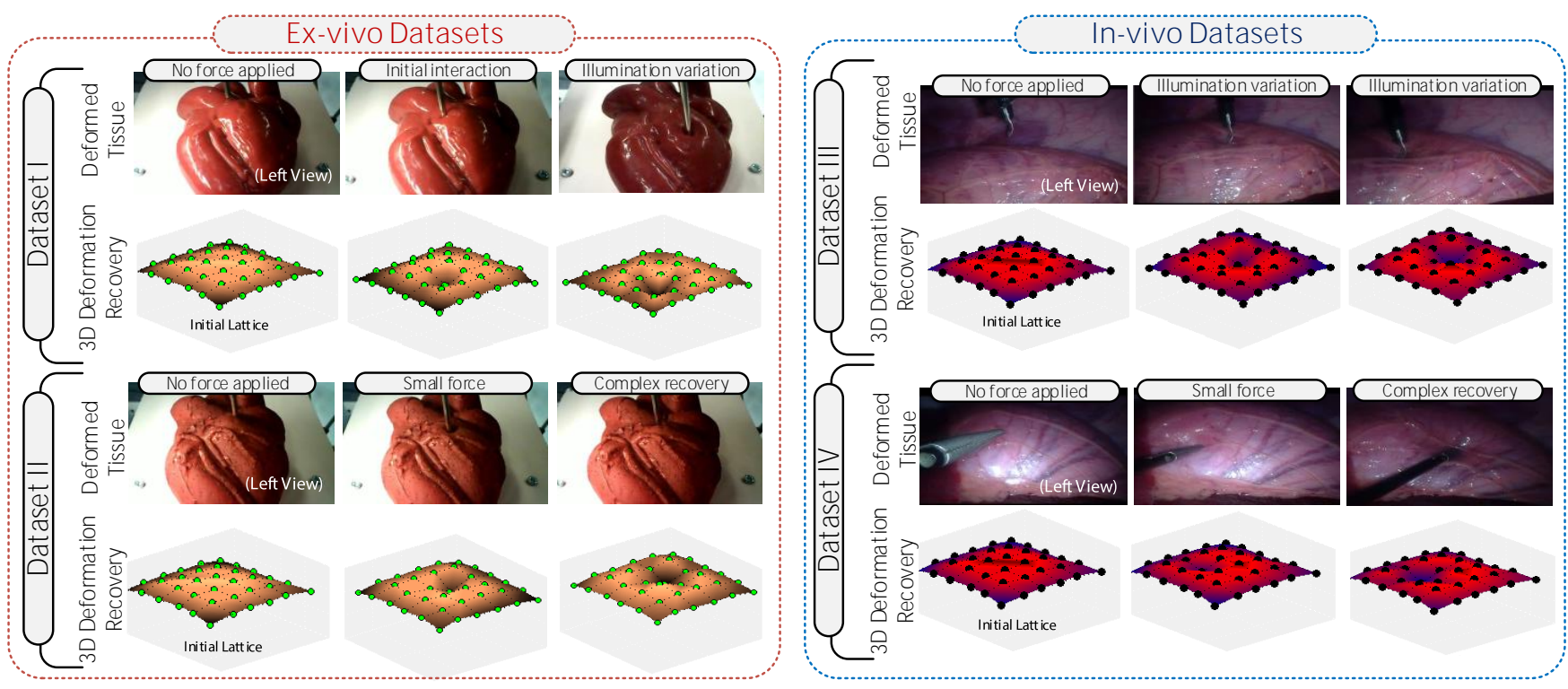

Fig. 5. Illustration of tissue deformations that result from applying force at different time instants, together with the 3D deformable structure recovered using our proposed visual approach. Our proposal was tested under different variation of illumination, occlusions and complex deformation.
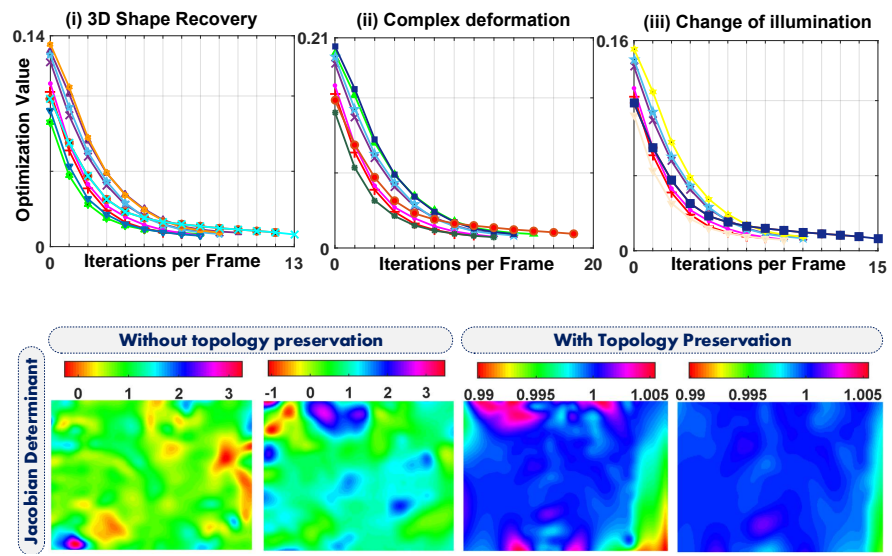

Fig. 6. (From top to bottom) Optimization plots resulted from our energy functional for different cases in which retrieving the 3D shape is challenging, including complex deformations and change of illumination. The Jacobian Determinant results of our vision based approach with and without applying our topology preservation term.

\section{EXPERIMENTAL RESULTS}

In this section, we describe the set of experiments we used to evaluate our proposed approach to perceive the interaction forces in robotic surgical systems.

\section{A. Data and Tasks Description}

We used both ex-vivo and in-vivo datasets (see bottom of Fig. 4) to evaluate our proposal.

The ex-vivo datasets (I and II) were acquired using an experimental setup composed of a stereo camera, artificial tissues, and a set of robot manipulators (Stäubli RX60B) (see top of Fig. 4). The two artificial hearts are used to imitate variations between two different subjects and were made of ECOFLEX 0030 which has mechanical properties similar to those of human tissues. We obtained two stereo-pair images sequences of size $640 \times 480$ during 2100 seconds.

The two in-vivo datasets on the other hand (III and IV) are from the Hamlyn Center Laparoscopic / Endoscopic Video library [23] and they both exhibit tool-tissue interaction and deformation. The sequences are composed of stereo-pair images of size $720 \times 288$ recorded along 450 seconds.

During the acquisition of the datasets, general palpation over the tissue was done. This is important since it allows for example identifying lesion and tumors, cutting tissue, and avoiding tissue penetration. In particular, the ex-vivo data palpation was carried out varying three main factors: position, orientation and illumination.

\section{B. Results}

To prove the benefits of our proposal, we offer detailed evaluation of both our diffeomorphic deformation mapping and the perception of the interaction force approaches. Fig. 5 shows the 3D diffeomorphic structure, bounded by our defined lattice, recovered using our proposed visual approach from the four different datasets. The tissues experience deformation from applying force over time and darker shades represent intense deformation at contact point. The plots clearly show pleasant visual results of the deformation field with both in-vivo and ex-vivo data in cases where recovering the $3 \mathrm{D}$ deformation is complicated including change of illumination and complex views. Apart from offering visual results, we also analyzed the convergence of our energy functional. The top part of Fig. 6 shows that the minimization of our functional, on different frames and for the different cases shown in Fig. 5, needed less than 20 iterations to get the minima.

This is further supported by the plots in Fig.7, taken from dataset $I$, in which the computed displacement at contact point (in $\mathrm{X}, \mathrm{Y}, \mathrm{Z}$ directions) are compared against 

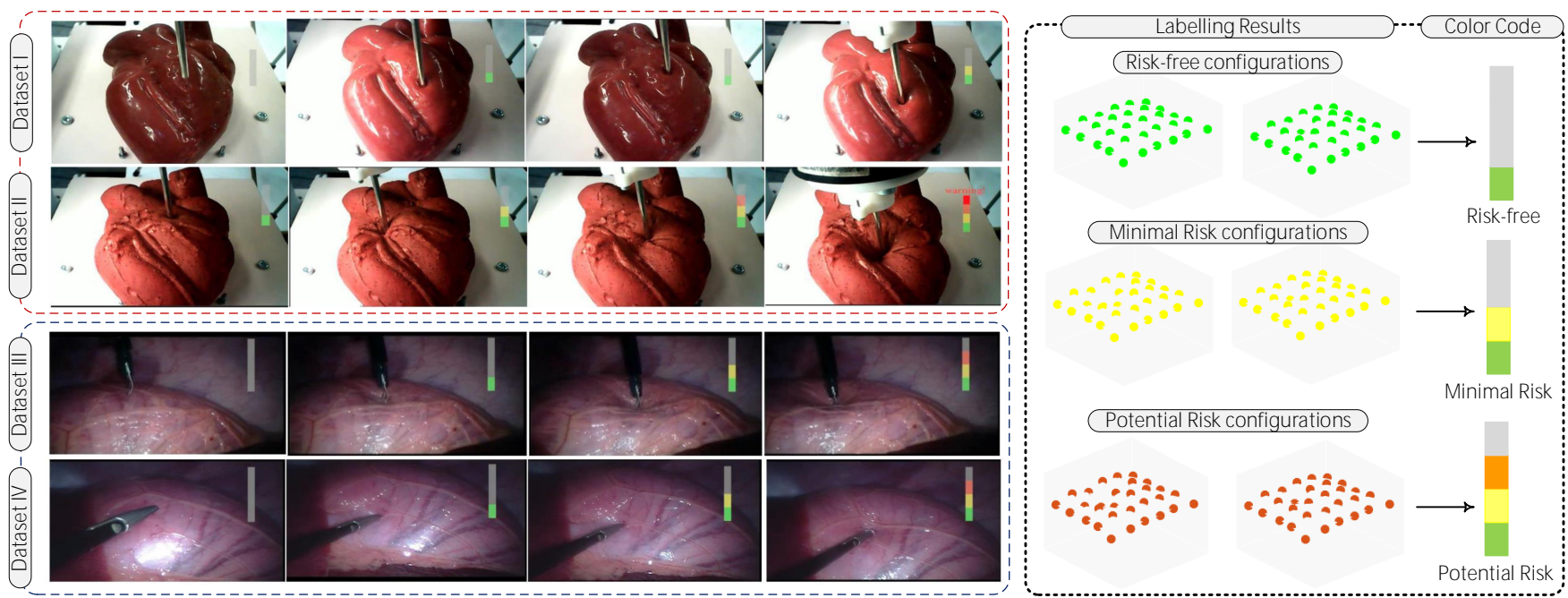

Fig. 7. Left side shows sample frames from the four datasets with the force feedback visual cue displayed. Right side shows the labeling results of some example cases in which the particular color code is assigned depending on the configuration.

TABLE I

NUMERICAL ANALYSIS OF OUR VISUAL BASED APPROACH WITH DIFFERENT DEGREES OF PENALIZATION $\mathbf{E}_{v p}$

\begin{tabular}{|c|c|c|c|c|c|}
\hline & Dataset I & Dataset II & Dataset III & Dataset IV & \\
\hline \multirow{2}{*}{ Exp. 1 - Eq. (2) with $\mathbf{E}_{v p}=0$} & $2.1399 e^{-3}$ & $5.2846 e^{-2}$ & $3.9488 e^{-3}$ & $2.4958 e^{-2}$ & Minimum \\
\hline & {$\left[\begin{array}{lll}-1.8468 & 2.9830\end{array}\right]$} & {$\left[\begin{array}{lll}-2.5960 & 3.0853\end{array}\right]$} & {$\left[\begin{array}{lll}-2.5960 & 3.0853\end{array}\right]$} & {$\left[\begin{array}{lll}-3.5893 & 2.0485\end{array}\right]$} & {$[\min , \max ]$ of $J_{\Phi}$} \\
\hline \multirow{2}{*}{ Exp. 2 - Eq. (4) with $\varphi=0$} & $4.3827 e^{-5}$ & $3.0834 e^{-6}$ & $6.3598 e^{-6}$ & $4.2358 e^{-5}$ & Minimum \\
\hline & {$\left[\begin{array}{lll}0.8882 & 1.1395\end{array}\right]$} & {$\left[\begin{array}{lll}0.9081 & 1.0932\end{array}\right]$} & [0.8452 1.1539] & {$\left[\begin{array}{lll}0.8283 & 1.1490\end{array}\right]$} & {$[\min , \max ]$ of $J_{\Phi}$} \\
\hline \multirow{2}{*}{ Exp. 3 - Eq. (4) with $\varphi=7 \cdot 10^{-2}$} & $1.9829 e^{-5}$ & $4.7694 e^{-6}$ & $5.4965 e^{-6}$ & $4.9357 e^{-6}$ & Minimum \\
\hline & {$\left[\begin{array}{lll}0.9081 & 1.004\end{array}\right]$} & {$\left[\begin{array}{lll}0.9248 & 1.0051\end{array}\right]$} & {$\left[\begin{array}{lll}0.9035 & 1.0094\end{array}\right]$} & {$\left[\begin{array}{lll}0.9248 & 1.006\end{array}\right]$} & {$[\min , \max ]$ of $J_{\Phi}$} \\
\hline
\end{tabular}

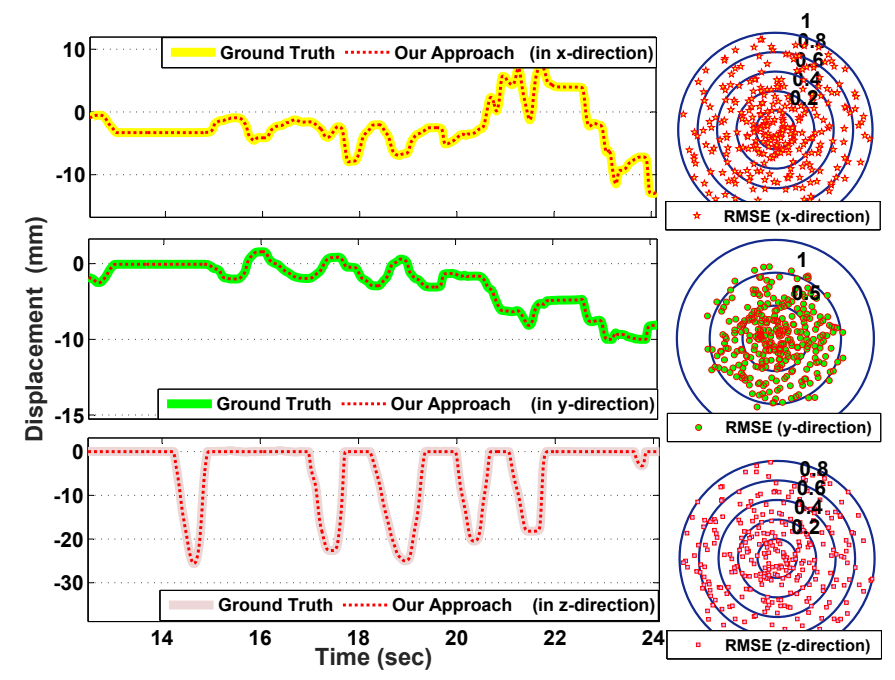

Fig. 8. Left side plots compare the computed displacement (at contact point) in $\mathrm{X}, \mathrm{Y}, \mathrm{Z}$ directions against the reference measurements given by the geometry of motion of the robot. Right side plots illustrate the RMSE results in all directions.

the reference measurement. By acquiring the geometry of motion from the robotic manipulator, we were able to obtain a ground truth reference of the interaction between the tool and the tissue. This point is a good reference for evaluating our approach since its recovery is very challenging due to the occlusions produced by the surgical tool. The results, left side of Fig. 8, show that the measurements are very close to each other. For a more quantitative analysis, we show the Root-Mean-Square Error (RMSE) at the right side of Fig. 8. The plots show a concentration of values lower than 1 $\mathrm{mm}$ in all directions. This demonstrates the accuracy of our computed measurements even during complex deformations.

For a detailed numerical analysis, we evaluate our energy functional, Eq. 3, using the four datasets described in Subsection III-A and the results are reported in Table 1. Exp. 1 shows that without a diffeomorphic deformation, the residuum is in the order of magnitude of $10^{-2}$ and $10^{-3}$ and has negative and big values of the Jacobian determinant. This indicates big expansions and contractions during the transformations which yields an unrealistic deformation.

In contrast, Exp. 2 and Exp. 3 reported a better minima in the order of magnitude of $10^{-5}$ and $10^{-6}$. A significant improvement is achieved in terms of the Jacobian determinat in which values are positives and close to 1 , which indicate preservation of region convexity of the mesh elements and, 
in consequence, preservation of the anatomy. A comparison between Exp. 2 and Exp. 3 shows that the second term of Eq. 4 allows better control of the magnitude of the expansions and contractions. It is worth mentioning that Exps. 2 and 3 allowed a $35 \%$ faster computation than Exp. 1. Some samples showing the Jacobian determinant over the region of interest are displayed at the bottom part of Fig. 6 .

Finally, taking into account that the ultimate goal of this work is to offer to the surgeon a visual stimulus by relating the deformation information into corresponding force feedback, we apply the generative labeling approach on the computed data and show the results in Fig. 7. The left side of the figure shows sample frames from the four different datasets with the visual cues for transmitting the force feedback. Furthermore, the right side shows some examples of the labeling results in which the data was accurately assigned. Qualitatively, our labeling approach had an average error of $2.05 \%$ for all datasets.

\section{CONCLUSION}

Surgeons who operate robotic surgical systems perform delicate surgical tasks remotely through the manipulators without directly interacting with the patients. This teleoperation setting eliminates the sense of touch and, in consequence, the perception of tool-tissue interaction forces. The force sensory substitution approach presented in this work offers a feasible alternative that overcomes this limitation and could potentially improve RAMIS performance. Our approach is based on a variational framework which allows computing the observable deformation. It allows recovering a realistic deformation while preserving the anatomical structure of the tissue. This information is then labeled to a corresponding applied force which is represented by a colorcode in a dynamic bar.

The experimental results verify that the extracted visualgeometric information are accurately mapped to the corresponding force level with an average labeling error of $2.05 \%$. Experiments included various datasets, both in-vivo and exvivo, and the computed results were validated against the ground truth obtained from the robotic manipulator. Our solution gave accurate results and offered a root-mean square error less than $1 \mathrm{~mm}$ in all directions.

Finally, this solution is promising in different clinical scenarios in which force has direct implication such as microsurgery, knot tying or needle-based procedures.

From a technical point of view, our aim is to establish a starting point for research in this area. Future work will include extensive clinical evaluation to measure the level of assistance this visual aid is providing to the surgeon.

\section{ACKNOWLEDGMENT}

This work was supported by a FPU national scholarship from the Spanish Ministry of Education with reference AP2012-1943. The work has been developed in the frame of Project RTC-2015-3926-1, from MINECO and with Feder funds.

\section{REFERENCES}

[1] E. Wilson, H. Bagshahi, and V. Woodruff, Overview of General Advantages, Limitations, and Strategies, Book Chapter, Robotics in General Surgery, Springer, 2014.

[2] N. Enayati, E. De Momi, and G. Ferrigno, Haptics in Robot-Assisted Surgery: Challenges and Benefits, IEEE Reviews in Biomedical Engineering Journal Vol. 9, pp. 49-65, 2016.

[3] G. Spinoglio, A. Marano, and F. G., Robotic Surgery: Current Applications and New Trends, Springer, 2015.

[4] T. Lendvay, B. Hannaford, and R. Satava, Future of Robotic Surgery, The Cancer Journal Vol. 19, No. 2, pp. 109-119, 2013.

[5] M. Yip, S. Yuen, and R. Howe, A Robust Uniaxial Force Sensor for Minimally Invasive Surgery, IEEE Transactions on Biomedical Engineering, Vol. 57, No. 5, pp. 1008-1011, 2010.

[6] P. Puangmali, H. Liu, K. Althoefer, and L. Seneviratne, Miniature 3Axis Distal Force Sensor for Minimally Invasive Surgical Palpation, IEEE Transactions on Mechatronics, pp. 646-656, 2012.

[7] A. Faragasso, J. Bimbo, Y. Noh, A. Jiang, S. Sareh, H. Liu, T. Nanayakkara, H. Wurdemann, and K. Althoefer, Novel Uniaxial Force Sensor based on Visual Information for Minimally Invasive Surgery, IEEE International Conference on Robotics and Automation, pp. 29342939,2014

[8] A. Okamura, L. Verner, C. Reiley, and M. Mahvash, Haptics for Robot-Assisted Minimally Invasive Surgery, Book Chapter in Robotics Research, Springer Tracts in Advanced Robotics, pp. 361-372, 2011.

[9] S. Sokhanvar, J. Dargahi, S. Najarian, and S. Arbatani, Clinical and Regulatory Challenges for Medical Devices, Tactile Sensing and Displays: Haptic Feedback for Minimally Invasive Surgery and Robotics, Wiley Publications, 2012.

[10] M. Greminger and B. Nelson, Vision-Based Force Measurement, IEEE Transactions on Pattern Analysis and Machine Intelligence, Vol.15, No. 4, pp. 290-298, 2004.

[11] J. Kim, F. Janabi-Sharifi, and J. Kim, A Haptic Interaction Method Using Visual Information and Physically Based Modeling, IEEE Transactions on Mechatronics, Vol. 15, No. 4, pp. 636-645, 2010.

[12] E. Noohi, S. Parastegari, and M. Zefran, Using Monocular Images to Estimate Interaction Forces During Minimally Invasive Surgery, IEEE International Conference on Intelligent Robots and Systems, 2014.

[13] W. Kim, S. Seung, K. Choi, S. Park, S. Ko, and J. Park, Image-Based Force Estimation of Deformable Tissue using Depth Map for SinglePort Surgical Robot, International Conference on Control, Automation and Systems, pp. 1716-1719, 2012.

[14] M. Greminger and B. Nelson, Modeling Elastic Objects with Neural Networks for Vision-Based Force Measurement, IEEE/RSJ Intl. Conference on Intelligent Robots and Systems, pp. 1278-1283, 2003.

[15] F. Karimirad, S. Chauhan, and B. Shirinzadeh, Vision-based Force Measurement using Neural Networks for Biological Cell Microinjection, Journal of Biomechanics, Volume 47, pp. 1157-1163, 2014.

[16] A.I Aviles, S.M. Alsaleh, J.K. Hahn and A. Casals, Towards Retrieving Force Feedback in Robotic-Assisted Surgery: A Supervised NeuroRecurrent-Vision Approach, IEEE Transactions on Haptics, 2016.

[17] A.I. Aviles, S.M. Alsaleh, P. Sobrevilla, and A. Casals, ForceFeedback Sensory Substitution using Supervised Recurrent Learning for Robotic-Assisted Surgery, Annual International Conference of the IEEE Engineering in Medicine and Biology Society (EMBC), 2015.

[18] P. Bach-y-Rita and S.W. Kercel, Sensory Substitution and the HumanMachine Interface, Trends in Cognitive Sciences Journal Vol.7, No.12, pp.541-546, 2003.

[19] B. Bethea, K. M. Okamura, A.M., T. Fitton, S. Cattaneo, V. Gott, W. Baumgartner, and Y. D.D., Application of Haptic Feedback to Robotic Surgery, Journal of Laparoendoscopic \& Advanced Surgical Techniques, 2004.

[20] M. Unser, Splines: A Perfect Fit for Signal and Image Processing, IEEE Signal Processing Magazine, vol.16, no.6, pp. 22-38, 1999.

[21] A.I. Aviles, T. Widlak, A. Casals and H. Ammari, Towards Estimating Cardiac Motion Using Low-Rank Representation and Topology Preservation for Ultrafast Ultrasound Data, Annual International Conference of the IEEE Engineering in Medicine and Biology Society, 2016.

[22] A.P. Dempster, N.M. Laird and D.B. Rubin , Maximum Likelihood from Incomplete Data via the EM Algorithm, Journal of the Royal Statistical Society, 1977.

[23] P. Mountney, D. Stoyanov and G.-Z. Yang, Three-Dimensional Tissue Deformation Recovery and Tracking: Introducing techniques based on laparoscopic or endoscopic images, IEEE Signal Processing Magazine, Volume: 27. Issue: 4. pp. 14-24, 2010. 